\title{
LA PLANIFICACIÓN DEL ENTRENAMIENTO DEPORTIVO: CAMBIOS VINCULADOS A LAS NUEVAS FORMAS DE ENTENDER LAS ESTRUCTURAS DEPORTIVAS CONTEMPORÁNEAS
}

JOSÉ M. GONZÁLEZ RAVÉ

FERNANDO J. NAVARRO VALDIVIELSO

PEDRO MIGUEL PEREIRA GASPAR

\section{Resumen}

El presente estúdio procura realizar un resumen sobre las modificaciones y tendencias en modelos de planificación de entrenamiento deportivo tradicionales e contemporaneos. Más concretamente si discute la utilización de nuevos conceptos complementares de modelos classicos de planificación y las modificaciones en las estruturas de competición actuales, bien como las mejorias en las condiciones materiales y la inversión necesária hasta el alto rendimiento. Los contributos de la ciencia a las adaptaciones a la carga de entrenamiento son factores sobre los quales és necesarios reflectir para una planificación eficaz del entrenamiento deportivo moderno.

\section{Palabras-Clave:}

Planificación; Programación; Periodización; Entrenamiento deportivo; Carga de entrenamiento.

\section{THE PLANNING OF THE SPORT TRAINING: TIE CHANGES TO THE NEW FORMS TO UNDERSTAND THE CONTEMPORARY SPORT STRUCTURES}

JOSÉ M. GONZÁLEZ RAVÉ

FERNANDO J. NAVARRO VALDIVIELSO

PEDRO MIGUEL PEREIRA GASPAR

\begin{abstract}
The present study tries to make a summary on the modifications and tendencies in the models of traditional and contemporary planning of the sport training. The use of new concepts that complement the classics models, the modifications in the structure of present competitions, the improvements in the material conditions, the necessary investment until the high performance, the contributos of science and the adaptations to the training load are all factors that are necessary for reflection for an effective planning of the modern sport training.
\end{abstract}

\section{Key-Words:}

Planning; Programming; Periodization: Sport training; Training load. 


\section{Introducion}

Planificar supone por una parte adoptar una serie de decisiones en torno al futuro que nosotros deseamos respecto a una serie de cuestiones referidas a la optimización del rendimiento deportivo. La planificación del entrenamiento deportivo se debe entender como un proceso con una serie de decisiones que debe tomar el entrenador respecto a la mejora y la eficacia del rendimiento de un deportista en función del contexto en el que nos veamos envueltos, iniciación, niveles bajos y medios de competición o alto rendimiento deportivo.

\section{Modificaciones Metodologicas en la Planificación del Entrenamiento Deportivo}

El entrenamiento deportivo ha sufrido en los últimos años una serie de modificaciones respecto a la periodización tradicional que solía utilizarse debido a una serie de causas como:

1. La modificación radical en las condiciones económicas, sociales, organizativas y profesionales en las que se ve inmerso el deportista de alto rendimiento.

2. Las actuales circunstancias obligan a un entrenamiento apoyado por la ciencia y a una búsqueda profunda de reservas para mejorar el rendimiento; necesidad de una cooperación entre entrenadores y científicos. La discusión bibliográfica se inició en Rusia (WERCHOSHANKSKIJ, 1998a; MATWEJEW, 1998; PLATANOV, 1998; SELUNOV, 1998; SUSLOV e FILIN, 1998) y posteriormente se encuentra en otras publicaciones europeas (ISSURIN e SHKLIAR, 2001; MATWEJEW, 2001; TSCHIENE, 1999; ZANON, 1999).

3. Mediante la periodización del entrenamiento deportivo (Matwejew, 1964) se formulan por un lado el fundamento metodológico de la estructuración del entrenamiento de los deportistas y, por otro lado, los conceptos terminológicos. Con razón este principio se podría denominar clásico y básico. Sin embargo, parece bastante obvio que las tesis que se han formulado hace más de 40 años necesitan volver a ser meditadas y reformadas.

4. Los nuevos y alternativos conceptos que de ello surgen no rechazan la teoría clásica, sino que la complementan.

Vamos a analizar cada una de ellas.

\section{Condiciones económicas, sociales, organizativas y profesionales}

Uno de los factores sobre el que se están modificando las programaciones del entrenamiento es la excesiva presencia de competiciones, si hay competiciones durante todo el año, ¿cómo puede el deportista rendir óptimamente los 365 días al año?, el deportista es un ser humano sometido a estímulos, 
denominados de cargas de entrenamiento, y estas tienen como finalidad obtener una o varias puestas en forma que se han basado fundamentalmente en dobles periodizaciones como el caso del atletismo, o en deportes colectivos mantener una forma constante, y solo en momento puntuales de la temporada alcanzar puntas de rendimiento, seguidas de bajadas profilácticas, por lo tanto competiciones todo el año no asegura un rendimiento óptimo durante todo ese periodo, al contrario, lo que se logra es disminuir el rendimiento (DIGEL, 2001).

Otro factor a considerar es la presencia de instalaciones adecuadas para los deportistas de Alto Rendimiento, ya sean estatales como en los casos europeos de Alemania, Italia o España, que se ocupan de la atención del deportista en todos sus aspectos, frente a este tenemos el modelo de EEUU en el que no existe un departamento gubernamental que se ocupe del deporte de alto rendimiento, ni un solo empleado, todo depende del sector privado, del Comité Olímpico y de una mentalidad empresarial (DIGEL, 2001).

Otro factor a considerar es la financiación, los contribuyentes norteamericanos no pagan sus impuestos al gobierno para que este dé dinero a los sistemas de deporte de alto rendimiento. Sin embargo, en la mayoría de sistemas se financia gracias a los contribuyentes. En el caso español existe un modelo de financiación mixta estado- empresas a través del denominado plan ADO. El funcionamiento de ADO es similar al de cualquier entidad privada, administrando y distribuyendo a los deportistas a través de las diferentes Federaciones Deportivas Nacionales las aportaciones económicas que proceden de los patrocinadores y entidades colaboradoras.

Asimismo, ADO cuenta con una estructura adecuada que se ocupa de las áreas propias de una empresa de gestión de patrocinio deportivo: administración, finanzas, marketing, comunicación, relaciones públicas, etc. Desde el área técnica, ADO mantiene un contacto constante con las diferentes Federaciones para estar al tanto de sus necesidades y ajustar las cantidades a recibir anualmente, procedentes de las empresas patrocinadoras.

\section{Entrenamiento apoyado por la ciencia producto de la discusión iniciada en los países del este de europa}

Toda esta discusión aparece reflejada sobre una serie de elementos teóricos que sirvieron para el desarrollo de una teoría del entrenamiento por parte de los autores clásicos como Matwejew o Zatziorski, 
que según Selujanov (1999) no consideraron los trabajos de Vorobiev sobre halterofilia, o de Bondarchuck sobre los lanzamientos, que contenían descripciones de estructuras de entrenamiento, que se basaban en principios teóricos orientados hacia la adaptación. Esto en opinión de Selujanov (1999) ha sido el gran error de Matwejew. Por otra parte, la periodización del entrenamiento de Matwejew ha sufrido críticas por parte de otros metodólogos del entrenamiento provenientes de la escuela soviética y de otros países del este. Verchoshanskij critica la periodización del entrenamiento, ya que, según él, es muy rudimentaria la técnica utilizada para estructurar el entrenamiento deportivo:

[...] esta es una combinación especulativa lineal de partes estandarizadas, a saber los típicos microciclos , que forman a modo de bloques de construccion de niños para colocar encima otros bloques un poco mas voluminosos denominados mesociclos, que a su vez configuran los grandes microciclos como la estructura de construcción mayor [...] (PLATONOV, 2000, p. 88).

aunque después esa critica la suaviza manifestando que el proceso, aunque pueda parecer analítico y separado entre los elementos que existen, el resultado final nos dice como son mucho más significativas las conexiones ocultas de estos elementos que se materializan en la continuidad y las interrelaciones de los efectos obtenidos. Todas estas críticas de Verchoshanskij, según Platonov (2000), no responden a una reflexión actualizada de los conocimientos en periodización actuales, sino que es una crítica a los postulados que se realizaron en los años 50 y que se han ido actualizando por parte de Matwejew (2001).

\section{Las tesis que se han formulado hace más de 40 años necesitan volver a ser meditadas y reformadas.}

Es cierto que este debate genera discusión acerca de cuestiones que en los años 60 y 70 tenían plena vigencia, en relación a los planteamientos teóricos que en esa época estaban vigentes, o dicho de otro modo, el modelo de periodización de Matwejew estaba enfocado a los principios, conceptos y práctica que correspondía al nivel de desarrollo de aquellos momentos. Aunque bien es cierto que Matwejew (2001) ha efectuado una serie de propuestas teóricas actualizadas respecto a su modelo defendiendo la validez de éste.

Los nuevos y alternativos conceptos que de ello surgen no rechazan la teoría clásica, sino que la complementan.

Matwejew (2001) alude a la formación de conceptos científicos sobre el entrenamiento contemporáneo y como estos han seguido dos tendencias: en primer lugar el cambio de unos conceptos analíticos y descriptivos de sus componentes hacia el estudio integral del entrenamiento prestando cada vez más atención al orden general de la evolución general del proceso de entrenamiento dentro de sus etapas, periodos y ciclos enteros. 
La segunda tendencia es el estudio de las correlaciones del entrenamiento deportivo anual de muchos años con otras esferas de la actividad del deportista, en el que el entrenamiento deportivo sobresale como uno de los subfactores (otros pueden ser el régimen general de vida, factores complementarios al entrenamiento, etc), como una parte más del conjunto de la preparación del deportista.

Donde atacan sus críticos hablando de sistematización, descripción excesiva y carente de sustrato científico, Matwejew (2001, p. 237) habla ahora de integral, pero atendiendo a la organización y sistematización, en este sentido habla del concepto construcción del entrenamiento como: “[...] ordenar consecutivamente todo lo que en él se hace en base a las regularidades de la estructuración y evolución del proceso de entrenamiento[...]", posición que puede complementarse con las tesis de Verchoshanskij en la que el proceso de entrenamiento no es una suma de partes individuales (microciclos) en cualquier distinta combinación, sino un todo formado por múltiples capas, cuya estructura está formada por partes (etapas). El contenido y la organización se entiende de forma procesual, determinados por objetivos, tareas y condiciones objetivas como la adaptación al trabajo muscular.

En este sentido, nuestro planteamiento en torno a esta cuestión se aborda desde una perspectiva científica y pragmática dotando de sentido a ambas estructuras de entrenamiento para que no sean encontradas y enfrentadas, sino complementarias y sinérgicas entre sí y adaptadas a las diversas posibilidades que la competición y el entrenamiento posee en la actualidad.

\section{La carga como reguladora de los modelos de entrenamiento}

Las cargas de entrenamiento provocan adaptaciones que podemos considerar agudas o de corto plazo, que provocan tras un tiempo adaptaciones crónicas generales por su propia acción específica hacia los órganos y estructuras del deportista (VIRU, 1995). La carga se explica como la cantidad de efectos que el esfuerzo tiene sobre el estado funcional del deportista. Zintl (1991, p. 12) la define como la totalidad de los estímulos efectuados sobre el organismo, o dicho de otro modo, el resultado de las exigencias presentadas al deportista. Es decir en un modelo biológico todo estímulo produce una respuesta, por lo tanto el estímulo es el ejercicio, y dicho ejercicio se denomina carga externa, mientras que la respuesta es la que el organismo produce para generar adaptación, denominada en el contexto de entrenamiento carga interna. 
Para Tschiene (1997), al seguir los modelos de adaptación del organismo sometido a cargas de entrenamiento, a corto y a largo plazo, es necesario un análisis y clasificación de las cargas de entrenamiento según las posibilidades de adaptación de aquel a éstas. En este sentido es el análisis de las cargas de entrenamiento las que nos van a dar una idea inicial del tipo de estructuras en base a las cargas de entrenamiento.

Por tanto nos encontramos con dos conceptos a analizar que nos darán una primera aproximación, adaptación y carga de entrenamiento.

Los componentes de la carga de entrenamiento se definen por un determinado número de caracteres que para Platonov (1995, p. 96) son:

1. naturaleza de los ejercicios;

2. intensidad del trabajo;

3. duración del trabajo;

4. duración y naturaleza de los intervalos de reposo situados entre los ejercicios;

5. número de repeticiones.

Zintl (1991, p. 12) a la hora de analizar las cargas de entrenamiento establece para el mismo los componentes vinculados a la magnitud de ésta, es decir, intensidad, duración, densidad, volumen y frecuencia de carga. 
En este sentido, la propuesta de análisis de cargas realizada por Navarro (2000) describe con mayor rigor un análisis más sistemático de la carga en base a una serie de parámetros que son los que muestran la Figura 1:

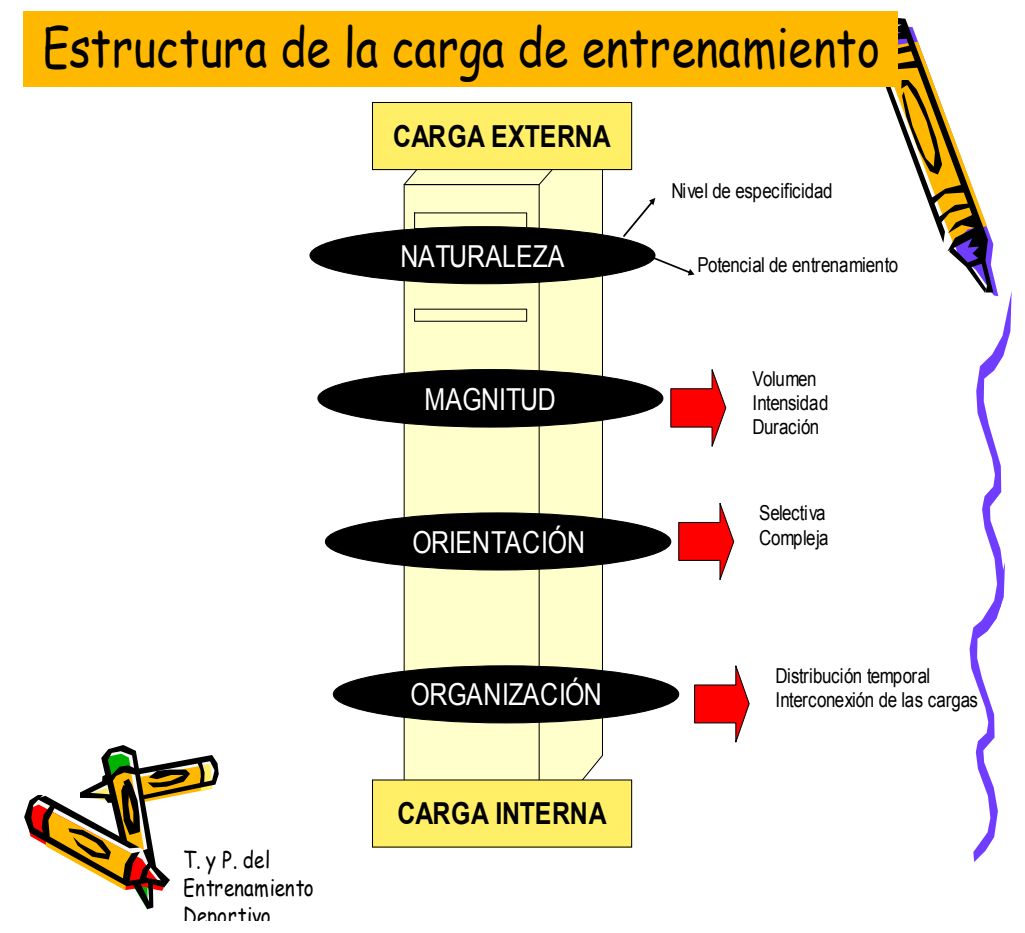

Figura 1: Estructura de la carga de entrenamiento.

Por otro lado, la carga de entrenamiento se encuentra condicionada por una serie de factores a la hora de diseñar su planificación como son: el deportista, la especialidad deportiva, y el tipo de competición en el que se va a situar cada uno de ellos.

Si hablamos de la naturaleza de la carga hablamos de lo que se va a trabajar y viene determinado por el nivel de especificidad, que indica la mayor o menor similitud del ejercicio con la manifestación propia de la competición. En este sentido la teoría clásica de Matwejew a dado más relevancia a la preparación general que lo que hacen los contemporáneos, que solo admiten este tipo de preparación general como medio de recuperación, como preparación de la carga de entrenamiento o como medio compensatorio (NAVARRO, 2000).

Para Navarro (2000), en la periodización clásica, los descriptores de la carga general y/o específica se basaban exclusivamente en la similitud de los ejercicios con el específico competitivo. Sin embargo, la especificidad de las reacciones del organismo a un estímulo de entrenamiento puede ser observado a 
través del trazo metabólico, por ejemplo, la acumulación de los productos de desecho del metabolismo que inducen inmediatamente la síntesis proteica después de un esfuerzo muscular. Por lo tanto el trazo metabólico se encuentra en relación a la adaptación que los estímulos provocan sobre el metabolismo, por lo que vamos a centrarnos en la adaptación como concepto que permite explicar los cambios a nivel metabólico y estructural.

\section{LAS ADAPTACIONES COMO RESULTADO-EFECTO DEL NIVEL DE ESPECIFICIDAD DE LA CARGA}

\section{La adaptación}

La adaptación se define como la modificación funcional y morfológica de los sistemas orgánicos frente a estímulos eficaces de carga. (ZINTL, 1991, p. 12). La adaptación como modificación a la reacción biológica producida por el ejercicio determinara si el entrenamiento produce adaptaciones orientadas hacia la mejora del rendimiento deportivo. Como ya hemos comentado anteriormente, las cargas de entrenamiento provocan adaptaciones agudas o de corto plazo, y tras un tiempo adaptaciones crónicas generales por su propia acción específica hacia los órganos y estructuras del deportista (VIRU, 1995).

Las adaptaciones a corto plazo han sido estudiadas por González Ravé (2005), donde se comprueba como solo con dos días de entrenamiento se consiguen adaptaciones que duran 9 días, este hallazgo permite comprobar como el entrenamiento de cargas concentradas en dos días, posee un efecto residual duradero que permite introducir contenidos de entrenamiento más específicos cuando nos encontramos próximos a una competición y dejar entrenamientos concentrados alejados de ésta para minimizar la fatiga. Por otro lado permite reducir al máximo de eficacia estos entrenamientos eliminando el entrenamiento "superfluo" tal y como afirma Starkey et al. (1994). Sin embargo estos efectos por su naturaleza aguda no generan cambios duraderos sobre el trazo metabólico del individuo. Hace falta una estimulación durante un determinado periodo de tiempo que genere adaptaciones crónicas, es decir que en función de la carga, la cantidad de ARNi (información sobre el aparato genético de las células) provoca una síntesis proteica que actúa sobre los músculos, sistema cardiovascular y respiratorio, así como al sistema endocrino para asegurar una especificidad de la síntesis proteica de los estímulos a los que se somete el deportista (TSCHIENE, 1997; NAVARRO, 2000). 


\section{Síntesis Proteica}

La síntesis proteica involucra principalmente a las proteínas necesarias para crear estructuras celulares activas y enzimas, que catalizarán las reacciones bioquímicas necesarias que determinan las funciones celulares específicas (VIRU, 1996) tal y como muestra la Figura 2.

Hablamos por tanto de procesos, basados en trabajos científicos que explican como un deportista pueda, y deba someterse, durante un tiempo prolongado a intensos esfuerzos de entrenamiento por medio de los cuales se realizan las modificaciones y adaptaciones fisiológicas bioquímicas y morfológicas, características de un deportista para la obtención de resultados (THEODORESCU,1997).

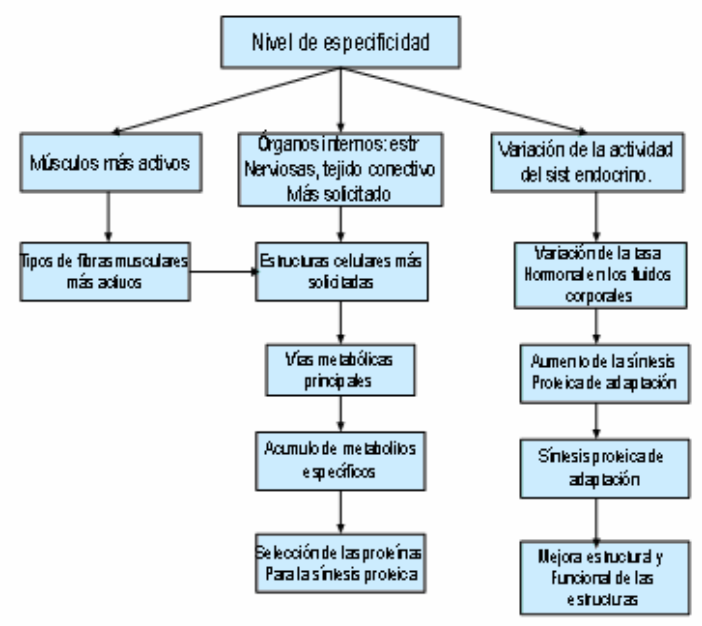

Figura 2: Esquema general de la carga sobre la estructura de entrenamiento. Fonte: VIRU, 1996, p. 9

\section{Potencial de entrenamiento}

El potencial de entrenamiento se define como la forma en que la carga estimula la condición del deportista. Se reduce con el incremento de la capacidad de rendimiento. Verchoshanskij y Siff (2000, p. 427) lo definen como la influencia de la carga sobre la forma física de los deportistas. Este concepto tiene especial relevancia en los modelos contemporáneos a través del potencial de adaptación del organismo Cuanto más alto sea el potencial entrenante relativo a la forma física actual del deportista, mayor será la probabilidad de aumentar la capacidad de trabajo especial del deportista (VERCHOSHANSKIJ ; SIFF, 2000). El potencial entrenante de los medios empleados decrece al mismo tiempo que se incrementa la capacidad de trabajo especial, por tanto es importante preservarlo mediante la introducción en el entrenamiento de medios más eficaces. (VERCHOSHANSKIJ; SIFF, 2000, p. 427) 


\subsection{Volumen de la carga}

El volumen de la carga se define como el aspecto cuantitativo de la carga con el que se pretende sumar todos los elementos homogéneos entre sí y reflejar así la cantidad total de actividad realizada por un atleta o equipo durante el entrenamiento (BOMPA 1983).

Verchoshanskij y Siff, (2000) se refieren a él como los aspectos cuantitativos del entrenamiento y desempeña un importante papel en la adaptación a largo plazo del cuerpo al trabajo muscular intenso, dicha conceptualización es debida a que estos autores consideran el volumen como un concepto que abarca la magnitud de la carga, su duración e intensidad, la magnitud de la carga es lo que habitualmente hemos denominado en nuestro contexto como volumen, como la medida cuantitativa planificada o real, que se calcula durante cada microciclo o estadios específicos o durante cada ciclo anual en conjunto Matveyev (1977). El volumen de entrenamiento ha aumentado notablemente en el entrenamiento contemporáneo, aunque un aumento demasiado grande de volumen en una sesión podría conllevar a una lesión o fatiga excesiva, por lo que es preferible aumentar el número de sesiones por microciclo cuando se estime lo suficiente el nivel de volumen (NAVARRO 2000).

\section{Intensidad}

La intensidad sería el aspecto cualitativo de la carga ejecutado en un periodo de tiempo (BOMPA,1983). La intensidad de la carga controla la potencia y la especificidad del estímulo sobre el organismo, así a más trabajo por unidad de tiempo mayor intensidad. Para Navarro (2000, p. 61);

\footnotetext{
la mayor intensificación del entrenamiento ha sido uno de los aspectos más característicos en el cambio de periodización clásica a los modelos actuales. En determinados periodos del ciclo anual se admite una intensificación de las cargas de entrenamiento, aunque solo después de una preparación preliminar, basada en una carga de volumen elevado pero de baja intensidad.
}

\section{Duración de la carga}

La duración de la carga es un elemento importantísimo sobre la dinámica del rendimiento de los deportistas Verchoshanskij y Siff (2000 p. 427). Hace referencia a los efectos que tiene el entrenamiento sobre la capacidad condicional que se va a estimular. Los efectos se refieren al periodo de influencia del estímulo o en un periodo más largo de cargas con una misma orientación.

La duración de la carga, se verá condicionada por la distribución de las cargas, ya sean regulares o concentradas, concepto que definiremos en el apartado de orientación de la carga. 


\section{Orientación de la carga}

La orientación de la carga está definida por la cualidad o capacidad que es potenciada y por la fuente energética solicitada. Está orientación puede ser clasificada en dos tipos:

- La cargas de tipo selectiva: cuando privilegia a una determinada capacidad.

- O también la carga puede ser compleja: cuando se solicitan diferentes capacidades y sistemas funcionales.

Las características de ambas se muestran en la Figura 3.

Sesión selectiva.

Sesión compleja.

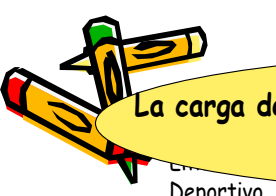

Figura 3: Características de la sesión compleja y selectiva.
-Mas posibilidades de concentración de carga con orientación especifica.

- Menor posibilidad de interacción negativa con

otros Objetivos.

- Mayor $n^{0}$ de sesiones/microciclo para satisfacer

las Necesidades de entrenamiento.

- Necesario seleccionar los objetivos atendiendo a

Su interacción.

- Necesario adecuar el orden de entrenamiento

con las diferentes orientaciones.

-Exige una mayor extensión de la sesión para

satisfacer el objetivo.

En el caso de las cargas complejas se han sugerido algunas alternativas de combinación de cargas en un orden tal que produzcan interacción positiva entre cargas dirigidas al desarrollo de la resistencia (VOLKOV, 1986 citado por NAVARRO, 2000):

- Aeróbico después de anaeróbico alactico;

- Aeróbico después de anaeróbico glucolítico (con bajo volumen);

- Anaeróbico glucolítico después de anaeróbico alactácido.

En estas condiciones, la carga de entrenamiento anterior crea las condiciones favorables para la carga sucesiva y para el aumento del efecto de la sesión de entrenamiento. 
La orientación selectiva en muy difícil de conseguir debido a que toda actividad física promueve una serie de mecanismos reguladores, por lo que parece más adecuado hablar del concepto de orientación privilegiada.

Los modelos contemporáneos utilizan en mayor medida las cargas selectivas que privilegian un determinado trabajo de una capacidad condicional, sobrecargando ese sistema metabólico y alcanzar posteriormente una respuesta adaptativa mayor.

Mientras que los modelos clásicos han optado en su mayor medida por sesiones de tipo complejo. En estos casos, la preocupación por conseguir efectos acumulativos de distintas orientaciones ha marco la pauta en la orientación de cargas, el problema es que se necesita mucho más plazo de tiempo para alcanzarlas.

\section{Organización de la carga}

El siguiente elemento de análisis de las cargas es la organización de ésta, que consiste en la sistematización de la carga en un periodo de tiempo dado con el fin de conseguir un efecto positivo acumulado de las cargas de diferente orientación.

En la organización distinguimos entre la distribución de las cargas en el tiempo y la interconexión de estas (VERCHOSHANSKIJ, SIFF, 2000).

La distribución de las cargas es la forma en que se colocan las diferentes cargas en una sesión, microciclo, mesociclo o macrociclo. La carga de este modo se puede repartir uniformemente en el ciclo (cargas regulares), o concentrarse en fases definidas del ciclo anual (cargas concentradas), tal y como muestra la Figura 4. 


\section{Cargas regulares.}

Contenidos de

entrenamiento

distribuidos en

más tiempo.

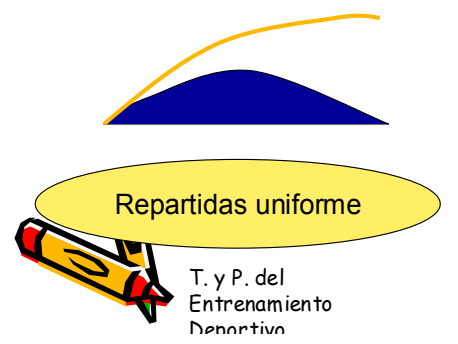

Figura 4: Distribución de cargas.

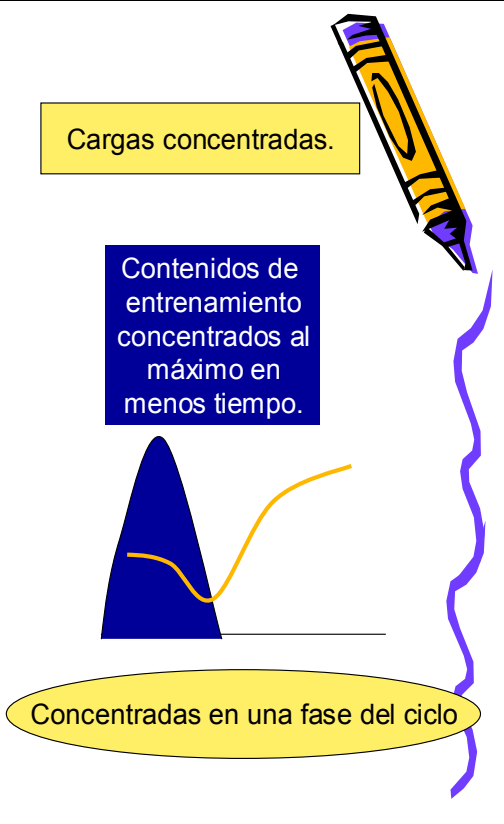

La teoría clásica aboga por una utilización de cargas regulares que provocan reacciones funcionales de corta duración, que no garantizan las condiciones para el desarrollo de los cambios de adaptación de larga duración en el organismo.

Las teorías contemporáneas utilizan preferentemente las cargas concentradas que proporcionan cambios funcionales más profundos en el rendimiento del deportista.

La interconexión de las cargas indica la relación que las cargas de diferente orientación tienen entre sí. Una combinación racional asegura un efecto acumulativo óptimo. El problema de la organización de las cargas está estrechamente ligado a la elección de un intervalo optimo de recuperación-estímulo de carga, y la alternancia de éstas.

Con el fin de obtener el rendimiento en las cargas en su interconexión se utilizan dos tipos de métodos, el método paralelo-complejo y el método secuencial, con el primero las cargas de trabajo tienen prioridades diferentes, y un efecto cuantitativo monótono, a pesar del aumento de volumen e intensidad. Al mismo tiempo, las reacciones del cuerpo a los componentes específicos son insignificantes.

Por otra parte el método secuencial introduce las cargas en forma de bloques, existiendo un orden sucesivo en éstas de manera que permitan adaptaciones morfológicas que favorecen los efectos deseados 
en la interconexión de cargas.

Una vez vistas las diferencias entre los modelos clásicos y contemporáneos vamos a continuación a describir cada una de las estructuras de entrenamiento referidas durante el texto empezando por el modelo de periodización de Matveyev, para continuar con los modelos más actuales como el modelo ATR y el de bloques de Verchoshanskij

\section{La Estructura Del Modelo Clásico De Matveyev}

Matveyev (1977) estructura su macrociclo en tres grandes periodos durante la temporada:

\section{Periodo Preparatorio}

2. Periodo Competitivo

\section{Periodo de Transición}

El periodo preparatorio se subdivide en fases de preparación general y especial. El propósito principal de preparación general es establecer la base funcional para el entrenamiento, por tanto provocamos una mejora de la condición física (NAVARRO, 2000. Aplicado a deportes con componentes técnicos, también debemos, aparte de elevar las posibilidades funcionales del organismo, en la técnica aplicar la gama de fundamentos y destrezas motoras, y en deportes de equipo en la estrategia-táctica hacer una introducción a los sistemas que se pretenden realizar y aspectos teóricos básicos del juego (HDEZ MORENO, 1988).

En la preparación especial los objetivos a conseguir en la preparación física se orientan hacia el desarrollo de las cualidades físicas específicas, se deben crear las bases de las capacidades motoras y técnicas de acuerdo a los requerimientos específicos de cada deporte. En cuanto a la técnica y la táctica en deportes de equipo empezamos a introducir dichas acciones en el contexto real. Los aspectos teóricos siguen la misma orientación (HDEZ MORENO, 1988).

El periodo competitivo debe ser subdividido en tres partes:

Fase de competiciones tempranas en la que se busca desarrollar las capacidades específicas motoras y técnicas, se busca una elevación del potencial del deportista para que pueda tomar parte en una serie de competiciones, pero sin llegar al máximo en ellas sino como preparatorias del siguiente periodo que es el de competiciones principales. 
La fase de competiciones principales es la parte más importante de la temporada, el entrenamiento en este periodo está caracterizado por la intensidad máxima, específica y similar a la competición, la parte principal de los ejercicios lo constituye el entrenamiento modelado. Puede haber competiciones previas a la importante.

La fase de competiciones finales no es una componente del macrociclo, pero puede aparecer debido a que el calendario de competiciones puede seguir tras la competición principal, se trata de mantener la forma en tanto en cuanto sigamos compitiendo pero teniendo en cuenta la fatiga de la fase principal.

El periodo de transición será un intervalo de descanso para establecer los medios de recuperación adecuados, para comenzar la siguiente temporada.

La periodización tradicional se puede establecer en base a uno, dos o tres picos de forma, teniendo en cuenta ciertos periodos competitivos en los que deseamos establecer una puesta a punto adecuada. Aunque las premisas en las que se basa este diseño hacen que solo se pueda establecer un pico de forma único, mientras que los otros son menores, tal como se muestra en la Figura 5.

Periodización de un ciclo

\begin{tabular}{|c|c|c|c|c|c|c|c|c|c|c|c|}
\hline 1 & II & III & IV & $V$ & VI & VII & VII & IX & $x$ & $x \mid$ & $x \| 1$ \\
\hline \multicolumn{12}{|c|}{\begin{tabular}{l|l} 
PPQ & XPE \\
\end{tabular}} \\
\hline \multicolumn{5}{|c|}{ Periodo preparatorio } & \multicolumn{5}{|c|}{ Perio do competitivo } & \multicolumn{2}{|l|}{$\begin{array}{l}\text { Periodo } \\
\text { Iransición }\end{array}$} \\
\hline \multicolumn{12}{|c|}{ Periodización de dos ciclos } \\
\hline Oct & Nov & Dic & Ene & Fet & Mar & Abril & Mayo & Jun & Jul & Agos & Sept \\
\hline \multicolumn{4}{|c|}{ Periodo preparatorio } & \multicolumn{2}{|c|}{$\begin{array}{c}\text { Periodo } \\
\text { competitivo }\end{array}$} & $\operatorname{man}$ & \multicolumn{2}{|c|}{$\begin{array}{c}\text { Periodo } \\
\text { Preparaterio II }\end{array}$} & \multicolumn{2}{|c|}{$\begin{array}{l}\text { Periodo } \\
\text { Competitivo II }\end{array}$} & $\begin{array}{l}\text { Per } \\
\text { Trans | }\end{array}$ \\
\hline \multicolumn{12}{|c|}{ Periodización de tres ciclos } \\
\hline Oct & Nov & Dic & Ene & $\mathrm{Fe}$ & Mar & Abril & Mayo & Jun & Jul & Agos & Sept \\
\hline \multicolumn{3}{|c|}{ Periodo preparatorio } & Period & \multicolumn{2}{|c|}{$\begin{array}{c}\text { Periodo } \\
\text { preparatorio }\end{array}$} & $\begin{array}{c}\text { Peri } \\
\text { comp }\end{array}$ & $\begin{array}{l}\text { odo } \\
\text { etitivo }\end{array}$ & \multicolumn{2}{|c|}{$\begin{array}{l}\text { Periodo } \\
\text { Preparatorio }\end{array}$} & $\begin{array}{l}\text { Periodo } \\
\text { Competitivo }\end{array}$ & $\begin{array}{l}\text { Per } \\
\text { Trans! }\end{array}$ \\
\hline
\end{tabular}

\section{Figura 5. Periodização clássica de Matveyev}

Fonte: GONZALEZ RAVE, J. M.

\section{EI Modelo de Macrociclo Contemporaneo}

La concentración de cargas permite emplear un entrenamiento altamente concentrado en lugar de un desarrollo complejo de muchas capacidades, este entrenamiento permite efectos más selectivos, inmediatos y acumulativos que utilizando el modelo convencional. 
Para Navarro (2000) la idea del concepto contemporáneo se basa en:

1. La concentración de cargas de entrenamiento sobre capacidades específicas u objetivos concretos de entrenamiento (capacidades/objetivos);

2. El desarrollo consecutivo de ciertas capacidades/objetivos en bloques de entrenamiento especializados o mesociclos.

En este modelo de entrenamiento concentrado el número de capacidades entrenables en un mesociclo debe disminuirse dejándolo en dos capacidades motoras y una característica de la técnica.

Por otra parte, las capacidades a trabajar deber ser establecidas de tal forma que se establezca un trabajo simultáneo entre cada mesociclo y teniendo en cuenta los efectos que se producen en él mediante la interacción de cargas (véase el apartado mesociclos).

El mesociclo de acumulación trata de elevar el potencial técnico y físico del deportista.

El mesociclo de transformación trata de transformar el potencial de las capacidades físicas y técnicas en preparación específica.

El mesociclo de realización trata de lograr resultados máximos dentro del trabajo realizado.

Esta ordenación de mesociclos, y en consecuencia de sus microciclos, se basa en el efecto acumulativo residual que ha producido el trabajo anterior, es decir el trabajo de acumulación debe mantenerse más tiempo que el trabajo de transformación y éste más tiempo que el de realización en relación a sus contenidos de entrenamiento y al tiempo de duración de los efectos que provoca. De este modo, cada mesociclo se basará en un trabajo que desarrolle las siguientes capacidades:

\section{MESOCICLO ACUMULACIÓN:}

- Entrenamiento aeróbico.

- Fuerza máxima.

\section{Mayor efecto residual.}

\section{MESOCICLO TRANSFORMACIÓN}

- Fuerza resistencia. 
- Capacidad glucolítica anaeróbica.

\section{Efecto residual medio.}

\section{MESOCICLO REALIZACIÓN}

- Entrenamiento anaeróbico aláctico.

- Entrenamiento en situaciones competitivas

- Táctica concreta.

\section{Efecto residual más pequeño.}

De este modo, los mesociclos discurren durante la temporada en forma de varios macrociclos que constituyen el modelo ATR, tal y como se puede ver en la figura 6 que mostramos a continuación.

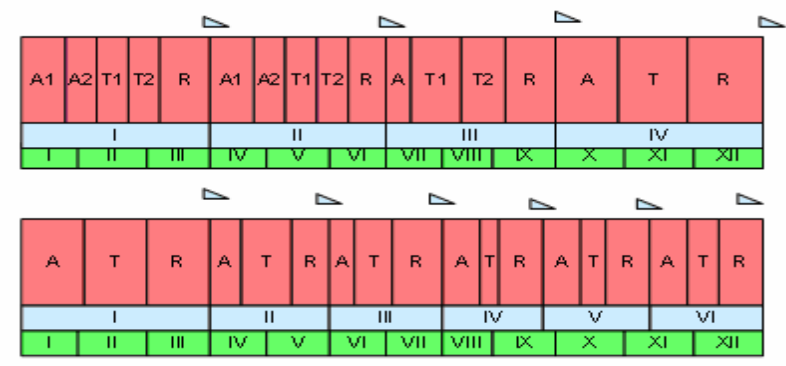

Figura 6. Periodização contemporánea ATR

Fonte: GONZALEZ RAVE, J. M.

La Figura 6 representa como en un ciclo anual existen varios macrociclos que discurren durante el año. Por otra parte, podemos obtener macrociclos más largo situando consecutivamente dos mesociclos de acumulación y dos macrociclos de transformación, logrando combinaciones precisas y más largas de entrenamiento.

En ese caso lo que debemos hacer es involucrar una capacidad a cada mesociclo, y aseguramos, el efecto residual por más tiempo.

Los tipos de entrenamiento predominantes si usamos consecutivamente varios mesociclos del mismo tipo podía ser esta a propuesta de Navarro (2000, p. 155): 


\begin{tabular}{lll} 
Quadro 1: Tipo de Mesociclos, tipo de treino principal e objectivos. \\
\hline MESOCICLOS & TIPO DE & OBJETIVOS \\
& ENTRENAMIENTO & ADICIONALES \\
\hline Acumulación 1 & Resistencia aeróbica & Fuerza máxima \\
Acumulación 2 & Fuerza máxima & Desarrollo general \\
& & Resistencia aeróbica \\
Transformación 1 & Fuerza resistencia & Desarrollo general \\
& & Resistencia \\
& & aeróbica- \\
Transformación 2 & Resistencia aeróbica- & Perfeccionamiento de la \\
& anaeróbica & técnica \\
& & Fuerza-resistencia \\
& & Resistencia anaeróbica \\
Realización & Perfeccionamiento de la \\
& Modelamiento de la & técnica \\
& competición. & Técnica \\
& Capacidades de velocidad & Táctica anaeróbica \\
\hline & &
\end{tabular}

Finalmente, las competiciones en este modelo se ubican preferentemente, en los mesociclos de realización, y siempre al final, de ahí que la situación de las competiciones condicione el diseño de planificación. Sin embargo, en caso de competiciones menores de preparación, estas pueden distribuirse en los mesociclos de realización o de transformación.

Por supuesto que el número de competiciones va a depender del tipo de deporte y su nivel de implantación y popularidad, y del nivel en el que se ubica el deportista, de tal manera que un deportista de élite debe atender a más competiciones, y estos deben tener entonces mayor número de microciclos.

\section{MODELO DE PLANIFICACIÓN DE VERCHOSHANSKIJ.}

No es hasta 1980 cuando Verchoshanskij establece su modelo de entrenamiento en bloques. La propuesta de cargas concentradas en bloques supuso que el trabajo "especial" como el lo denomina (VERCHOSHANSKIJ, 1996) se ampara en las siguientes premisas:

- El fín no solo debe el desarrollo de la fuerza muscular sino el aumento del potencial energético del cuerpo y de la capacidad para emplearlo en condiciones específicas de competición; 
- La mejora debe ser estructural abarcando todas las propiedades musculares (contráctiles, oxidativas y elásticas) que, según la especificidad del deporte favorecerán un aumento de fuerza máxima, explosiva o resistencia de fuerza.

Aplicado a la organización de los macrociclos de Verchoshanskij (1996) se establece como la concentración de cargas de trabajo reduce los parámetros funcionales de la capacidad de trabajo, lo que a su vez impide la mejora de la técnica y la velocidad de movimiento. Pero es un fenómeno de carácter temporal ya que cuando éstas se reducen aparece el efecto retardado del entrenamiento y a largo plazo produce un aumento de los parámetros funcionales

"Por consiguiente, las cargas concentradas de entrenamiento de preparación especial y las cargas dirigidas a mejorar la técnica o la velocidad de ejecución del ejercicio específico en competición, no se deben aplicar simultáneamente[...]" (VERCHOSHANSKIJ, 1996, p. 132).

Por otro lado en deportistas de élite es muy conveniente usarlo, para conseguir aumentar más su rendimiento específico, ya que cargas regulares no estimulan tanto a deportistas con años de entrenamiento a sus espaldas.

El macrociclo de Verchoshanskij (1996) abarca tres fases independientes (Figura 7):

- Fase preparatoria: tiene como fin predominante el desarrollar, mediante una preparación física especial, el potencial motor del atleta que es prerrequisito para el trabajo preferente a la velocidad de ejecución del ejercicio específico de competición.

- Fase especial: va dirigida a mejorar la capacidad del atleta para realizar el ejercicio de competición a una velocidad alta (potencia) imitando las condiciones (cargas) de competición.

- Fase de competición el objetivo es conseguir la velocidad más alta posible de ejecución del ejercicio de competición y de fortalecer las destrezas motoras del atleta.

Lo que tratamos es de conseguir una preparación multifuncional del atleta, para posteriormente perfeccionarla, y finalmente modelarla a las condiciones de competición y obtener la máxima velocidad. 
MACROCICLO DE VERCHOSHANSKIJ,

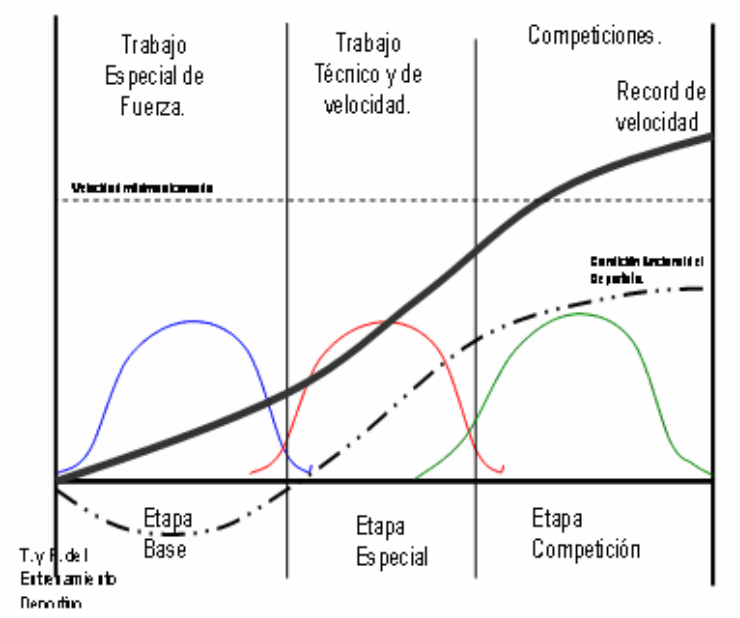

Figura 7: Periodização contemporánea de Verchoshanskij

Fonte: VERCHOSHANSKIJ, 1996, p. 135

\section{CONCLUSIÓN}

El propósito principal de este articulo fué mostrar de modo escueto y resumido cuales son las principales tendencias en el entrenamiento realizadas con deportistas. Practicamente la utilización de los modelos clasicos y contemporaneos aquí expuestos han mostrado resultados eficaces en la mejora del rendimiento deportivo, usando uno solo de ellos, o los dos a la vez, combinandolos cada uno con unos tipos de sujetos muy determinados, un deporte concreto y un modelos de competición muy específico. Lo importante en la utilización de cada uno de estos modelos es que constituyan un elemento que ayude a proporcionar a entrenadores respuestas para obtener el máximo rendimiento en sus deportistas.

Pese a que ahora surgen propuestas como modelos mátemáticos basados en ecuaciones que determinan el potencial biologico de desgaste y orientación de las carga, o modelos de predicción basados en sistemas complejos, todavía se encuentran muy alejados de la realizadad práctica que da respuestas concretas al entrenamiento deportivo.

\section{Referências Bibliográficas}

BOMPA, T. Theory and methodology of training. New York: Kendall/Hunt. 1983 
DIGEL H. Relaciones deportista-federación-centro. In: FORUM INTERNACIONAL DEL DEPORTE DE ELITE, 2. 2001. Sant Cugat. ANAIS ... CAR Sant Cugat, 2001, p. 116-147

GONZÁLEZ RAVÉ, J. M. Respuestas agudas al entrenamiento de fuerza máxima en deportistas femeninas. Archivos Medicina del Deporte, 2006 (admitido y en prensa).

MATVEYEV, L. P. Periodización del entrenamiento deportivo. Madrid: INEF, 1977

MATWEJEW, L. P. Teoría general del entrenamiento deportivo. Barcelona: Paidotribo, 2001.

NAVARRO, F. La controversia actual sobre la planificación del entrenamiento. INFOCOES, v. 5, n. 2, p. 55-69, 2000.

NAVARRO, F. Principios del entrenamiento y estructuras de la planificación deportiva. Modulo 2.1.1. Master en ARD. UAM-COE. 2000

PLATONOV, V. N. El entrenamiento deportivo, teoría y metodología. 4. ed. Barcelona: Paidotribo. 1995 PLATONOV, V. N. El concepto de la periodización del entrenamiento y el desarrollo de una teoría del entrenamiento. INFOCOES, v. 5, n. 1, p. 87-93, 2000

SELUJANOV, V. N. La clasificación científica-histórica del concepto de la periodización del entrenamiento y su crítica. INFOCOES, v. 4, n. 1, p. 89-91, 1999.

STARKEY, D. B. et. al. . One set of strength training is as good as three. Med. Sci. Sport Exerc. V. 26. n. 5, 1994. supl. 651.

THEODORESCU, L. El entrenamiento diario fraccionado. Rev. Entrenamiento Deportivo, v. 10, n. 4. p. 19-24, 1997.

TSCHIENE, P. Teoría del entrenamiento: clasificación de las cargas y modelos de los métodos de entrenamiento según el criterio de adaptación. INFOCOES, v. 2, n. 1 p. 74-83, 1997.

VERCHOSHANSKIJ, Y. Principios para una organización racional del proceso de entrenamiento dirigido a desarrollar la velocidad. Cuadernos de Atletismo, v. 37, p. 127-137, 1996.

. SIFF, M. Superentrenamiento. Barcelona: Paidotribo, 2000.

VIRU, A. Mecanismos de adaptación biológica y entrenamiento. Rev. Entrenamiento Deportivo, v. 9, n. 2, p. 6-11, 1996.

ZINTL, F. Entrenamiento de la resistencia. Barcelona: Martínez Roca, 1991. 
JOSÉ M. GONZÁLEZ RAVÉ

UNIVERSIDAD DE CASTILLA LA MANCHA

FACULTAD DE CIENCIAS DE LA ACTIVIDAD FÍSICA Y EL DEPORTE

FERNANDO J. NAVARRO VALDIVIELSO

UNIVERSIDAD DE CASTILLA LA MANCHA

FACULTAD DE CIENCIAS DE LA ACTIVIDAD FÍSICA Y EL DEPORTE

PEDRO MIGUEL PEREIRA GASPAR

UNIVERSIDADE DE COIMBRA

FACULDADE DE CIÊNCIAS DO DESPORTO E EDUCAÇÃO FÍSICA 